\title{
FOTOPERÍODO, TEMPERATURA E REGULADORES VEGETAIS NA GERMINAÇÃO DE SEMENTES DE Passiflora cincinnata Mast. ${ }^{1}$
}

\author{
VALDIR ZUCARELI², GISELA FERREIRA², AMANDA CRISTINA ESTEVES AMARO², \\ FRANCISCO PINHEIRO DE ARAÚJO²
}

\begin{abstract}
RESUMO - A espécie Passiflora cincinnata Mast., pertencente à família Passifloraceae, é silvestre e popularmente conhecida como maracujá-do-mato, sendo considerada importante na produção de porta-enxertos, uma vez que é tolerante à seca, a doenças causadas por bactérias e a nematóides, além de poder ser utilizada em programas de melhoramento genético. $\mathrm{O}$ trabalho teve como objetivos estudar o efeito da luz e da temperatura e a interação entre temperatura e reguladores vegetais na germinação de sementes de Passiflora cincinnata Mast. Foi constituído de três experimentos: no primeiro, estudou-se o efeito da luz e da temperatura na germinação de sementes; no segundo, o efeito de diferentes concentrações dos reguladores vegetais $\mathrm{GA}_{4+7}+\mathrm{N}$-(fenilmetil)-aminopurina na germinação das sementes e, no terceiro, a interação entre temperatura e reguladores vegetais na germinação das sementes. O delineamento experimental foi inteiramente casualizado para todos os experimentos e os dados foram submetidos à análise de variância e comparação das médias pelo teste Tukey, a 5\% de probabilidade. É possível observar que a luz exerce efeito inibitório sobre a germinação das sementes, e que os reguladores vegetais, $\mathrm{GA}_{4+7}+\mathrm{N}$-(fenilmetil)-aminopurina, são eficientes na superação da dormência, além de ampliarem os limites de temperatura da germinação. A temperatura alternada $20-30^{\circ} \mathrm{C}$ mostra-se a mais adequada para a germinação de sementes dessa espécie.
\end{abstract}

Termos para indexação: maracujá, passifloráceas, propagação.

\section{PHOTOPERIOD, TEMPERATURE AND PLANT GROWTH REGULATORS ON GERMINATION OF Passiflora cincinnata SEEDS}

\begin{abstract}
The species Passiflora cincinnata Mast., which belongs to the Passifloraceae family, is wild and commonly known as Crato Passion Fruit and it is also considered potentially important for rootstock production due to its tolerance to drought, bacterial diseases and nematodes. It can also be used in genetic breeding programs. This objective of this research was to study the effect of light and temperature and the interaction between temperature and plant growth regulators, on Passiflora cincinnata Mast. seed germination. Three experiments were developed: the first verified the effect of light and temperature on seed germination; the second, the effect of different concentrations of $\mathrm{GA}_{4+7}$
\end{abstract}

${ }^{1}$ Submetido em 20/08/2008. Aceito para publicação em 04/04/2009. Parte da dissertação de Mestrado do primeiro autor apresentada à Unesp.

${ }^{2}$ Universidade Estadual Paulista, Instituto de Biociências, Departamento de Botânica, Caixa Postal 510, 18618-000, Botucatu-SP, Brasil. valdirzucareli@yahoo.com.br, gisela@ibb.unesp.br, amandaamaro@uol. com.br

${ }^{3}$ Embrapa Semi-Árido, C.P. 23, 56302-970, Petrolina-PE, Brasil. Pinheiro@cpatsa.embrapa.br 


\begin{abstract}
+ $\mathrm{N}$-(phenylmethyl)-1H-purine-6-amine plant growth regulators on seed germination; and the third, the interaction between temperature and plant growth regulators on seed germination. A randomized complete design was used in all the experiments and data were subjected to analysis of variance followed by mean comparison by the Tukey test, at 0.05 significance. Light had an inhibitory effect on seed germination, and $\mathrm{GA}_{4+7}+\mathrm{N}$-(phenylmethyl)-1H-purine-6-amine were efficient in breaking dormancy and increasing temperature limits. Alternating temperatures, $20-30^{\circ} \mathrm{C}$, were the most appropriate for seed germination in this species.
\end{abstract}

Index terms: passion fruit, Passifloraceae, propagation.

\section{INTRODUÇÃO}

A espécie Passiflora cincinnata Mast., pertencente à família Passifloraceae, é silvestre (Aponte e Jáuregui, 2004), conhecida como maracujá-do-mato, sendo considerada importante na produção de porta-enxertos, uma vez que é tolerante à seca (Araújo et al., 2004), a doenças causadas por bactérias (São José, 1994; Meletti et al., 2002) e a nematóides, além de poder ser utilizada em programas de melhoramento genético (Ferreira e Oliveira, 1991).

A maioria dos problemas ocorridos no emprego de sementes de Passifloráceas na produção de mudas pé-franco ou para porta-enxertos está relacionados à heterogeneidade das mudas e a baixa porcentagem de germinação (MorleyBunker, 1980; Ruggiero, 1991).

Em sementes de Passiflora cincinnata, Lombardi (2003) relatou que essas apresentam baixa germinação (52\%) e, segundo Meletti et al. (2002), as sementes dessa espécie necessitam de tempo de armazenamento superior a dois anos para superação de sua dormência.

A luz constitui fator de suma importância na germinação das sementes e sobrevivência das plântulas. As espécies respondem de maneira diferente a sensibilidade à luz, existindo sementes cuja germinação pode ser influenciada, positivamente ou negativamente, pela luz e sementes que são indiferentes a ela (Borges e Rena, 1993). A temperatura apresenta, também, grande influência na germinação, atuando na porcentagem e na velocidade de germinação, pois afeta a absorção de água e as reações bioquímicas que regulam o metabolismo envolvido nesse processo, sendo que cada espécie possui uma faixa característica de temperatura dentro da qual suas sementes podem germinar (Bewley e Black, 1994).

Quando o desempenho das sementes é analisado sob algum tipo de estresse ambiental, a presença de reguladores vegetais deve ser considerada, devido à sua interferência na germinabilidade das sementes (Botelho e Perez, 2001).

Dessa forma, o presente trabalho teve como objetivo estudar o efeito da luz, da temperatura e a interação entre temperatura e os reguladores vegetais $\mathrm{GA}_{4+7}+$ $\mathrm{N}$-(fenilmetil)-aminopurina na germinação das sementes de Passiflora cincinnata.

\section{MATERIAL E MÉTODOS}

As sementes foram obtidas junto ao Centro de Pesquisa Agropecuária do Trópico Semi-Árido (CPATSA) Embrapa, sendo os frutos coletados de diversas matrizes no Campo Experimental da Caatinga, Petrolina-PE, no ano de 2004. Após a extração das sementes, essas foram lavadas para retirada da mucilagem e colocadas ao sol para secar por três dias. Em seguida à secagem, as sementes foram armazenadas em câmara fria com temperatura média de $10^{\circ} \mathrm{C}$, durante um ano. Por ocasião da implantação dos experimentos, foi determinado o grau de umidade das sementes pelo método da estufa a $105^{\circ} \mathrm{C} \pm 3$ por 24 horas (Brasil, 1992), o que resultou em $9 \%$ de água.

Para atingir os objetivos propostos, o trabalho foi subdividido em três experimentos. O primeiro foi instalado em delineamento experimental inteiramente casualizado, seguindo um esquema fatorial $9 \times 2$ (temperatura $\mathrm{x}$ fotoperíodo), sendo utilizadas cinco repetições de 25 sementes por parcela. Os tratamentos constituíram-se pela combinação entre nove temperaturas $\left(10^{\circ} \mathrm{C}, 15^{\circ} \mathrm{C}, 20^{\circ} \mathrm{C}\right.$, $25^{\circ} \mathrm{C}, 30^{\circ} \mathrm{C}, 35^{\circ} \mathrm{C}, 40^{\circ} \mathrm{C}, 20-30^{\circ} \mathrm{Ce} 30-20^{\circ} \mathrm{C}$ ) e duas condições de fotoperíodo (oito horas de luz e escuro constante). Nos tratamentos com alternância de temperatura $\left(20-30^{\circ} \mathrm{C}\right.$ e $30-20^{\circ} \mathrm{C}$ ) foi utilizado período de 16 horas para a primeira temperatura e oito horas para a segunda temperatura de cada regime de alternância, respectivamente. Para simular 
a ausência de luz, foram utilizadas caixas de acrílico, tipo gerbox, de coloração preta e, nos tratamentos submetidos à fotoperíodo de 8 horas de luz e 16 horas de escuro foram utilizados caixas de acrílico, tipo gerbox, transparente.

$\mathrm{O}$ segundo experimento foi implantado em delineamento experimental inteiramente casualizado com seis tratamentos de cinco repetições e 25 sementes por parcela. Os tratamentos foram constituídos pela testemunha (sementes embebidas em água) e pelas concentrações de 100, 200, 300, 400 e 500mg.L.-1 de GA ${ }_{4+7}+\mathrm{N}$-(fenilmetil)aminopurina. As sementes permaneceram imersas nas respectivas soluções durante cinco horas, sob aeração constante. Como fonte de reguladores vegetais, foi utilizado o produto comercial Promalin ${ }^{\circledR}$, composto por $1,8 \%$ de $\mathrm{GA}_{4+7}$ e 1,8\% N-(fenilmetil)-1H-6-aminopurina. Após a embebição, as sementes foram transferidas para caixas de acrílico, tipo gerbox, de coloração preta. As caixas foram mantidas em câmara de germinação sob temperatura alternada $20-30^{\circ} \mathrm{C}$ (16-8 horas).

A instalação do terceiro experimento foi realizada em delineamento experimental inteiramente casualizado num esquema fatorial $9 \times 2$ (temperatura $\times$ concentração) com cinco repetições de 25 sementes por parcela, sendo os tratamentos constituídos pela combinação entre nove condições de temperatura $\left(10^{\circ} \mathrm{C}, 15^{\circ} \mathrm{C}, 20^{\circ} \mathrm{C}, 25^{\circ} \mathrm{C}, 30^{\circ} \mathrm{C}\right.$, $35^{\circ} \mathrm{C}, 40^{\circ} \mathrm{C}, 20-30^{\circ} \mathrm{C}$ e $\left.30-20^{\circ} \mathrm{C}\right)$ e duas condições de reguladores vegetais: testemunha (sementes embebidas em água) e a concentração de $400 \mathrm{mg} \cdot \mathrm{L}^{-1}$ de $\mathrm{GA}_{4+7}+$ $\mathrm{N}$-(fenilmetil)-aminopurina). As sementes foram colocadas para germinar em caixas plásticas, tipo gerbox de coloração preta.

Nos três experimentos a semeadura foi realizada sobre duas folhas de papel mata-borrão, umedecidas com água destilada na proporção de duas vezes e meia a massa do papel (Brasil, 1992) e as caixas foram mantidas em câmaras de germinação. A contagem do número de sementes germinadas foi realizada diariamente durante 40 dias, sempre em sala de segurança com luz verde, sendo consideradas germinadas as sementes que apresentaram raiz primária com aproximadamente $2 \mathrm{~mm}$ de comprimento (Hadas, 1976).

Ao final de cada experimento foram determinadas as porcentagens de germinação (equivalente à somatória das porcentagens de plântulas normais e de plântulas anormais), de sementes mortas, de sementes dormentes, de plântulas normais e de plântulas anormais (Brasil, 1992), além do tempo médio de germinação (Labouriau, 1983) e índice de velocidade de germinação (Silva e Nakagawa,
1995). As porcentagens de sementes mortas e dormentes foram obtidas mediante teste do tetrazólio (Malavasi et al., 2001).

Os dados foram submetidos à análise de variância, e as médias comparadas pelo teste Tukey, a $5 \%$ de probabilidade (Pimentel Gomes, 1990).

\section{RESULTADOS E DISCUSSÃO}

De modo geral, a porcentagem de germinação foi baixa, independentemente da temperatura e condição de luz utilizada, atingindo o maior valor $(14,4 \%)$ no tratamento com alternância de temperatura $\left(20-30^{\circ} \mathrm{C}\right)$ sob escuro constante, o que não diferiu significativamente dos tratamentos $30-20^{\circ} \mathrm{C}(12,8 \%) \mathrm{e}$ $30^{\circ} \mathrm{C}(11,2 \%)$, ambos também no escuro (Tabela 1$)$.

Para Meletti et al. (2002) e Lombardi (2003), a baixa germinação obtida com sementes da espécie Passiflora cincinnata Mast. está relacionada ao elevado nível de dormência.

Resultados semelhantes foram observados em sementes de Passiflora alata Dryander e Passiflora edulis Sims, com diferenças significativas na germinação com o uso de temperatura alternada $20-30^{\circ} \mathrm{C}$ (Osipi e Nakagawa, 2005; Santos et al., 1999).

Os tratamentos que permaneceram sob ausência de luz apresentaram maiores médias de germinação, diferindo significativamente dos demais, demonstrando o efeito inibitório da luz. Esses resultados estão de acordo com o recomendado pelas Regras para Análise de Sementes (Brasil, 1992) para a espécie Passiflora edulis, o qual indica a realização do teste de germinação no escuro.

Até 40 dias de avaliação, não foram observadas plântulas anormais, provavelmente devido à baixa germinação e à não ocorrência de germinação em temperaturas extremas, o que poderia levar à formação de plântulas anormais devido à condição de estresse.

Não houve diferença significativa no tempo médio de germinação entre as temperaturas de $30^{\circ} \mathrm{C}, 20-30^{\circ} \mathrm{C}$ e $30-$ $20^{\circ} \mathrm{C}$, na ausência de luz. Entretanto, os tratamentos $20-30^{\circ} \mathrm{C}$ e $30-20^{\circ} \mathrm{C}$ apresentaram os maiores índices de velocidade de germinação, diferindo significativamente da temperatura de $30^{\circ} \mathrm{C}$. Resultados semelhantes foram obtidos na germinação de sementes de Passiflora giberti N.E. Brown, por Duarte Filho et al. (2000), os quais observaram maior uniformidade de germinação empregando temperatura alternada $30-20^{\circ} \mathrm{C}$.

A temperatura de $40^{\circ} \mathrm{C}$ foi nociva às sementes, promovendo a maior porcentagem de sementes mortas, independentemente da condição de luz. As sementes 
submetidas às temperaturas de $35^{\circ} \mathrm{C}$ e $40^{\circ} \mathrm{C}$ apresentaram incidência de fungos, o que corrobora com relatos de Ferraz Grande e Takaki (2006) de que sementes incubadas acima da temperatura máxima extravasam substâncias para o meio, possibilitando o desenvolvimento de microrganismos como fungos e bactérias, indicando sua deterioração. Observou-se também que, nas temperaturas de $10^{\circ} \mathrm{C}$ a $35^{\circ} \mathrm{C}$ as sementes permaneceram viáveis, porém dormentes.

TABELA 1. Germinação, tempo médio de germinação (tempo), índice de velocidade de germinação (IVG), porcentagem de sementes mortas e sementes dormentes de Passiflora cincinnata Mast. em função das temperaturas e condições de fotoperíodo.

\begin{tabular}{|c|c|c|c|c|c|c|c|c|c|c|}
\hline \multirow[b]{2}{*}{ Temperatura } & \multicolumn{2}{|c|}{$\begin{array}{c}\text { Germinação } \\
(\%)\end{array}$} & \multicolumn{2}{|c|}{$\begin{array}{c}\text { Tempo } \\
(\%)\end{array}$} & \multicolumn{2}{|c|}{ IVG } & \multicolumn{2}{|c|}{$\begin{array}{c}\text { Sementes Mortas } \\
(\%)\end{array}$} & \multicolumn{2}{|c|}{$\begin{array}{l}\text { Sementes Dormentes } \\
\text { (\%) }\end{array}$} \\
\hline & Luz & Escuro & Luz & Escuro & Luz & Escuro & Luz & Escuro & Luz & Escuro \\
\hline $10^{\circ} \mathrm{C}$ & $0,0 \mathrm{~A} \mathrm{~b}$ & $0,0 \mathrm{~A} \mathrm{c}$ & $0,0 \mathrm{~A} \mathrm{~b}$ & $0,0 \mathrm{~A} \mathrm{~b}$ & $0,0 \mathrm{~A} \mathrm{a}$ & $0,0 \mathrm{~A} \mathrm{c}$ & $12,0 \mathrm{~A} \mathrm{~d}$ & $16,8 \mathrm{~A} \mathrm{c}$ & $88,0 \mathrm{~A}$ a & $83,2 \mathrm{~A} \mathrm{a}$ \\
\hline $15^{\circ} \mathrm{C}$ & $0,0 \mathrm{~A} \mathrm{~b}$ & $0,0 \mathrm{~A} \mathrm{c}$ & $0,0 \mathrm{~A} \mathrm{~b}$ & $0,0 \mathrm{~A} \mathrm{~b}$ & $0,0 \mathrm{~A} \mathrm{a}$ & $0,0 \mathrm{~A} \mathrm{c}$ & $14,4 \mathrm{~A} \mathrm{~cd}$ & $17,6 \mathrm{~A} \mathrm{c}$ & $85,6 \mathrm{~A}$ ab & $82,4 \mathrm{~A} \mathrm{a}$ \\
\hline $20^{\circ} \mathrm{C}$ & $0,0 \mathrm{~A} \mathrm{~b}$ & $0,0 \mathrm{~A} \mathrm{c}$ & $0,0 \mathrm{~A} \mathrm{~b}$ & $0,0 \mathrm{~A} \mathrm{~b}$ & $0,0 \mathrm{~A} \mathrm{a}$ & $0,0 \mathrm{~A} \mathrm{c}$ & $24,8 \mathrm{~A} \mathrm{bcd}$ & $25,6 \mathrm{~A} \mathrm{bc}$ & $75,2 \mathrm{~A} a b c$ & $74,4 \mathrm{~A} \mathrm{ab}$ \\
\hline $25^{\circ} \mathrm{C}$ & $0,0 \mathrm{~A} \mathrm{~b}$ & $0,0 \mathrm{~A} \mathrm{c}$ & $0,0 \mathrm{~A} \mathrm{~b}$ & $0,0 \mathrm{~A} \mathrm{~b}$ & $0,0 \mathrm{~A} \mathrm{a}$ & $0,0 \mathrm{Acc}$ & $30,4 \mathrm{~A} b c$ & $31,2 \mathrm{~A} \mathrm{bc}$ & $69,6 \mathrm{~A} \mathrm{bc}$ & $68,8 \mathrm{~A} \mathrm{ab}$ \\
\hline $30^{\circ} \mathrm{C}$ & $1,6 \mathrm{~B}$ ab & $11,2 \mathrm{~A} \mathrm{a}$ & $6,4 \mathrm{~B}$ ab & $17,31 \mathrm{~A} \mathrm{a}$ & $0,4 \mathrm{~B} \mathrm{a}$ & $5,8 \mathrm{~A} \mathrm{~b}$ & $26,4 \mathrm{~B}$ bcd & $44,0 \mathrm{~A} \mathrm{~b}$ & $72,0 \mathrm{~A} a b c$ & $44,8 \mathrm{~B} \mathrm{c}$ \\
\hline $35^{\circ} \mathrm{C}$ & $0,0 \mathrm{~B} \mathrm{~b}$ & $4,0 \mathrm{~A} \mathrm{~b}$ & $0,0 \mathrm{~B} \mathrm{~b}$ & $16,0 \mathrm{~A} \mathrm{a}$ & $0,0 \mathrm{~A} \mathrm{a}$ & $0,0 \mathrm{~A} \mathrm{c}$ & $31,2 \mathrm{~A} \mathrm{bc}$ & $38,4 \mathrm{~A} \mathrm{~b}$ & $68,8 \mathrm{~A} \mathrm{bc}$ & $57,6 \mathrm{~A} \mathrm{bc}$ \\
\hline $40^{\circ} \mathrm{C}$ & $0,0 \mathrm{~A} \mathrm{~b}$ & $0,0 \mathrm{~A} \mathrm{c}$ & $0,0 \mathrm{~A} \mathrm{~b}$ & $0 \mathrm{~A} \mathrm{~b}$ & $0,0 \mathrm{~A} \mathrm{a}$ & $0,0 \mathrm{~A} \mathrm{c}$ & $99,2 \mathrm{~A} \mathrm{a}$ & $98,4 \mathrm{~A}$ a & $0,8 \mathrm{~A} \mathrm{~d}$ & $1,6 \mathrm{~A} \mathrm{~d}$ \\
\hline $20-30^{\circ} \mathrm{C}$ & $3,2 \mathrm{Ba}$ & $14,4 \mathrm{~A} \mathrm{a}$ & $18,3 \mathrm{~A} \mathrm{a}$ & $15,8 \mathrm{~A} \mathrm{a}$ & $0,5 \mathrm{~B} \mathrm{a}$ & $11,4 \mathrm{~A} \mathrm{a}$ & $25,6 \mathrm{~B} \mathrm{bcd}$ & $42,4 \mathrm{~A} \mathrm{~b}$ & $71,2 \mathrm{~A}$ abc & $43,2 \mathrm{~B} \mathrm{c}$ \\
\hline $30-20^{\circ} \mathrm{C}$ & $3,2 \mathrm{~B} \mathrm{a}$ & $12,8 \mathrm{~A} \mathrm{a}$ & $6,0 \mathrm{~B} \mathrm{ab}$ & $12,4 \mathrm{~A} \mathrm{a}$ & $3,3 \mathrm{~B} \mathrm{a}$ & $11,4 \mathrm{~A} \mathrm{ab}$ & $33,6 \mathrm{~A} \mathrm{~b}$ & $27,2 \mathrm{~A} \mathrm{bc}$ & $63,2 \mathrm{~A} \mathrm{c}$ & $60,0 \mathrm{~A} \mathrm{bc}$ \\
\hline \multicolumn{11}{|l|}{$\mathrm{F}$} \\
\hline Fotoperíodo & \multicolumn{2}{|c|}{$62,41 * *$} & \multicolumn{2}{|c|}{$14,90 * *$} & \multicolumn{2}{|c|}{$29,44 * *$} & \multicolumn{2}{|c|}{$5,44^{*}$} & \multicolumn{2}{|c|}{$16,84 * *$} \\
\hline Temperatura & \multicolumn{2}{|c|}{$31,84^{* * *}$} & \multicolumn{2}{|c|}{$14,86^{* *}$} & \multicolumn{2}{|c|}{$17,24 * *$} & \multicolumn{2}{|c|}{$103,86 * *$} & \multicolumn{2}{|c|}{$99,87^{* *}$} \\
\hline Fot.xTemp. & \multicolumn{2}{|c|}{$10,19^{* *}$} & \multicolumn{2}{|c|}{$3,49 * *$} & \multicolumn{2}{|c|}{$7,99 * *$} & \multicolumn{2}{|c|}{$1,83 \mathrm{~ns}$} & \multicolumn{2}{|c|}{$3,27 * *$} \\
\hline C.V. $(\%)$ & \multicolumn{2}{|c|}{72,99} & \multicolumn{2}{|c|}{60,16} & \multicolumn{2}{|c|}{46,82} & \multicolumn{2}{|c|}{15,94} & \multicolumn{2}{|c|}{11,85} \\
\hline
\end{tabular}

Médias seguidas de mesma letra, minúsculas na coluna e maiúsculas na linha, não diferem entre si pelo Teste Tukey a $5 \%$ de probabilidade.

* significativo a $5 \%$ de probabilidade.

** significativo a $1 \%$ de probabilidade.

Ns: não significativo a $5 \%$ de probabilidade.

$\mathrm{O}$ uso dos reguladores vegetais $\mathrm{GA}_{4+7}+\mathrm{N}$-(fenilmetil)aminopurina foi eficiente na superação da dormência das sementes, sendo observado aumento na porcentagem de germinação até a concentração de $300 \mathrm{mg} . \mathrm{L}^{-1}$ (Tabela 2), comportamento inverso foi observado para a porcentagem de sementes dormentes.

A efetividade dos reguladores vegetais, $\mathrm{GA}_{4+7}+$ $\mathrm{N}$-(fenilmetil)-aminopurina, na superação da dormência de sementes, também foi relatada por Ferrari (2005) em sementes de Passiflora alata Curtis, a qual obteve maiores médias de germinação na concentração de $250 \mathrm{mg}$. $\mathrm{L}^{-1}$.

Resultados discordantes são mencionados por Zucareli et al. (2003) que obtiveram maiores porcentagens de sementes dormentes nos tratamentos que continham fenilmetil- aminopurina (citocinina).

$\mathrm{Na}$ avaliação da porcentagem de plântulas normais, a maior média $(78,4 \%)$ foi observada na concentração de $300 \mathrm{mg} . \mathrm{L}^{-1}(78,4 \%)$, contudo não diferiu estatisticamente das concentrações de 400 e $500 \mathrm{mg} . \mathrm{L}^{-1}$, com $76,8 \%$ e $75,2 \%$, respectivamente.

Para plântulas anormais, as maiores médias foram observadas nas concentrações 400 e $500 \mathrm{mg} \cdot \mathrm{L}^{-1}$ de $\mathrm{GA}_{4+7}+$ $\mathrm{N}$-(fenilmetil)-aminopurina, 7,2\% e 5,6\% respectivamente, diferindo significativamente do tratamento testemunha, o qual não promoveu a formação de plântulas anormais. Resultados diferentes foram observados por Ferrari (2005), que não obteve plântulas anormais na germinação de sementes de Passiflora alata Curtis. Não foram observadas diferenças 
significativas para porcentagem de sementes mortas e para tempo médio de germinação.

As concentrações de 200, 300, 400 e 500mg. $\mathrm{L}^{-1}$ de $\mathrm{GA}_{4+7}+\mathrm{N}$-(fenilmetil)-aminopurina promoveram os maiores índices de velocidade de germinação $(17,71 ; 18,42 ; 26,14$ e 25,34; respectivamente). Concordando com os resultados observados por Ferrari (2005) em sementes de Passiflora alata Curtis, que obteve maiores índices de velocidade com uso de 200 e 250mg.L $\mathrm{L}^{-1}$ de $\mathrm{GA}_{4+7}+\mathrm{N}$-(fenilmetil)aminopurina.

TABELA 2. Germinação, plântulas normais, plântulas anormais, sementes dormentes, sementes mortas, tempo médio (tempo) e índice de velocidade de germinação (IVG) de sementes de Passiflora cincinnata Mast. tratadas com $\mathrm{GA}_{4+7}+\mathrm{N}$-(fenilmetil)-aminopurina.

\begin{tabular}{|c|c|c|c|c|c|c|c|}
\hline \multirow[b]{2}{*}{$\begin{array}{c}\text { Concentração } \\
\left(\mathrm{mg} . \mathrm{L}^{-1}\right)\end{array}$} & \multirow[b]{2}{*}{$\begin{array}{c}\text { Germinação } \\
(\%)\end{array}$} & \multicolumn{2}{|c|}{ Plântulas } & \multicolumn{2}{|c|}{ Sementes } & \multirow[b]{2}{*}{$\begin{array}{c}\text { Tempo } \\
\text { (dias) }\end{array}$} & \multirow[b]{2}{*}{ IVG } \\
\hline & & $\begin{array}{c}\text { Normais } \\
(\%)\end{array}$ & $\begin{array}{c}\text { Anormais } \\
(\%)\end{array}$ & $\begin{array}{c}\text { Dormentes } \\
(\%)\end{array}$ & $\begin{array}{c}\text { Mortas } \\
(\%)\end{array}$ & & \\
\hline 0 & $19,2 \mathrm{~d}$ & $19,2 \mathrm{~d}$ & $0 \mathrm{~b}$ & $73,6 \mathrm{a}$ & $7,2 \mathrm{a}$ & $16,2 \mathrm{a}$ & $4,50 \mathrm{c}$ \\
\hline 100 & $53,6 \mathrm{c}$ & $51,2 \mathrm{c}$ & $2,4 a b$ & $40,8 \mathrm{~b}$ & $5,6 \mathrm{a}$ & $14,4 \mathrm{a}$ & $13,37 \mathrm{~b}$ \\
\hline 200 & $62,4 \mathrm{bc}$ & $60 \mathrm{bc}$ & $2,4 \mathrm{ab}$ & $31,2 \mathrm{~b}$ & $6,4 \mathrm{a}$ & $13,4 \mathrm{a}$ & $17,71 \mathrm{ab}$ \\
\hline 300 & $80,8 \mathrm{ab}$ & $78,4 \mathrm{a}$ & $2,4 a b$ & $12,8 \mathrm{c}$ & $6,4 \mathrm{a}$ & $14,6 \mathrm{a}$ & $18,42 \mathrm{ab}$ \\
\hline 400 & $84,0 \mathrm{a}$ & $76,8 \mathrm{a}$ & $7,2 \mathrm{a}$ & $8,0 \mathrm{c}$ & $6,4 \mathrm{a}$ & $12,2 \mathrm{a}$ & $26,14 \mathrm{a}$ \\
\hline 500 & $80,6 \mathrm{ab}$ & $75 \mathrm{ab}$ & $5,6 \mathrm{a}$ & $7,2 \mathrm{c}$ & $11,2 \mathrm{a}$ & $13,8 \mathrm{a}$ & $25,34 \mathrm{a}$ \\
\hline $\mathrm{F}$ & $33,3 * *$ & $29,1 * *$ & $3,55^{*}$ & $41,8^{* * *}$ & $0,74 n s$ & $1,20 \mathrm{~ns}$ & $12,36^{* *}$ \\
\hline C.V. (\%) & 11,5 & 11,5 & 79 & 19,7 & 49 & 9,7 & 17,9 \\
\hline
\end{tabular}

Médias seguidas de mesma letra, na coluna, não diferem entre si pelo Teste Tukey a $5 \%$ de probabilidade.

* significativo a $5 \%$ de probabilidade.

** significativo a $1 \%$ de probabilidade.

Ns: não significativo a $5 \%$ de probabilidade.

Com base nos resultados obtidos nos dois primeiros experimentos foi realizado o terceiro experimento, no qual foi adotada a concentração de $400 \mathrm{mg} \cdot \mathrm{L}^{-1}$ de $\mathrm{GA}_{4+7}$ $+\mathrm{N}$-(fenilmetil)-aminopurina e ausência de luz para a germinação.

Houve interação entre temperaturas e reguladores vegetais em todos os parâmetros avaliados (Tabela 3). As sementes não tratadas com os reguladores apresentaram as maiores médias de germinação na temperatura de $30^{\circ} \mathrm{C}$ e nos regimes de alternância de temperaturas $\left(20-30^{\circ} \mathrm{C}\right.$ e 30 $20^{\circ} \mathrm{C}$ ). Mas com a adição dos reguladores houve estímulo significativo à germinação e aumento dos limites de tolerância à temperatura, sendo observada a germinação nas temperaturas entre 15 e $35^{\circ} \mathrm{C}$, incluindo-se as alternadas.

Observou-se que sementes não tratadas com reguladores e submetidas à temperatura de $25^{\circ} \mathrm{C}$ apresentaram apenas
2,4\% de germinação. Entretanto, quando tratadas com 400mg.L $\mathrm{L}^{-1}$ de $\mathrm{GA}_{4+7}+\mathrm{N}$-(fenilmetil)-aminopurina e submetidas à mesma temperatura, estas apresentaram porcentagem de germinação significativamente maior $(54,4 \%)$. Esses resultados estão de acordo com Cunha e Casali (1989) e Botelho e Perez (2001), os quais relatam a interferência dos reguladores vegetais na germinação de sementes, quando estas são submetidas a condições adversas, como o estresse térmico e hídrico. A ampliação dos limites de temperatura na germinação das sementes de maracujá, obtidas nesse trabalho, poderá facilitar a disponibilidade de mudas da espécie ao longo do ano.

Sementes tratadas com 400mg.L-1 de $\mathrm{GA}_{4+7}+$ $\mathrm{N}$-(fenilmetil)-aminopurina e submetidas à temperatura alternada, $20-30^{\circ} \mathrm{C}$, apresentaram as maiores médias na porcentagem de germinação $(66 \%)$, não diferindo 
significativamente da temperatura alternada $30-20^{\circ} \mathrm{C}$ $(49,6 \%)$, conforme Tabela 3 . O favorecimento da temperatura alternada na germinação de passifloráceas também foi mencionado por Santos et al. (1999) em sementes de Passiflora edulis f. flavicarpa Degener, por Duarte Filho et al. (2000) em sementes de Passiflora giberti N.E. Brown e por Osipi e Nakagawa (2005) em sementes de Passiflora alata Dryander.

TABELA3. Germinação, índice de velocidade de germinação (IVG), tempo médio de germinação (tempo), porcentagem de plântulas normais e anormais de Passiflora cincinnata Mast. em função da temperatura e do prétratamento com $\mathrm{GA}_{4+7}+\mathrm{N}$-(fenilmetil)-aminopurina.

\begin{tabular}{|c|c|c|c|c|c|c|c|c|c|c|}
\hline \multirow[b]{2}{*}{ Temperatura } & \multicolumn{2}{|c|}{$\begin{array}{c}\text { Germinação } \\
(\%)\end{array}$} & \multicolumn{2}{|c|}{ IVG } & \multicolumn{2}{|c|}{$\begin{array}{l}\text { Tempo } \\
(\%)\end{array}$} & \multicolumn{2}{|c|}{$\begin{array}{c}\text { Plântulas Normais } \\
\qquad \%)\end{array}$} & \multicolumn{2}{|c|}{$\begin{array}{c}\text { Plântulas Anormais } \\
\qquad \%)\end{array}$} \\
\hline & Água & $400 \mathrm{mg} \cdot \mathrm{L}^{-1}$ & Água & $400 \mathrm{mg} \cdot \mathrm{L}^{-1}$ & Água & $400 \mathrm{mg} \cdot \mathrm{L}^{-1}$ & Água & $400 \mathrm{mg} \cdot \mathrm{L}^{-1}$ & Água & $400 \mathrm{mg} \cdot \mathrm{L}^{-1}$ \\
\hline $10^{\circ} \mathrm{C}$ & $0,0 \mathrm{~A} \mathrm{c}$ & $0,0 \mathrm{~A} \mathrm{e}$ & $0,0 \mathrm{~A} \mathrm{~b}$ & $0,0 \mathrm{Ad}$ & $0,0 \mathrm{~A} \mathrm{~b}$ & $0,0 \mathrm{~A} \mathrm{c}$ & $0,0 \mathrm{~A} \mathrm{~d}$ & $0,0 \mathrm{Ad}$ & $0,0 \mathrm{~A} \mathrm{~b}$ & $0,0 \mathrm{~A} \mathrm{e}$ \\
\hline $15^{\circ} \mathrm{C}$ & $0,0 \mathrm{~B} \mathrm{c}$ & 29,6 A c & $0,0 \mathrm{~B} \mathrm{~b}$ & $6,5 \mathrm{Ac}$ & $0,0 \mathrm{~B} \mathrm{~b}$ & $27,0 \mathrm{~A} \mathrm{a}$ & $0,0 \mathrm{~B} \mathrm{~d}$ & $28,0 \mathrm{~A} \mathrm{c}$ & $0,0 \mathrm{~A} \mathrm{~b}$ & $1,6 \mathrm{Ade}$ \\
\hline $20^{\circ} \mathrm{C}$ & $1,6 \mathrm{~B} \mathrm{c}$ & $44,8 \mathrm{~A} \mathrm{bc}$ & $0,0 \mathrm{~B} \mathrm{~b}$ & $20,3 \mathrm{~A} a b$ & $5,2 \mathrm{~B} \mathrm{~b}$ & $13,6 \mathrm{~A} \mathrm{~b}$ & $1,6 \mathrm{~B} \mathrm{~cd}$ & $42,4 \mathrm{~A}$ abc & $0,0 \mathrm{~B} \mathrm{~b}$ & 2,4 A cde \\
\hline $25^{\circ} \mathrm{C}$ & $2,4 \mathrm{bc}$ & $54,4 \mathrm{~A} \mathrm{ab}$ & $0,2 \mathrm{~B} \mathrm{~b}$ & $21,7 \mathrm{~A} a b$ & $6,2 \mathrm{~B} \mathrm{~b}$ & $10,1 \mathrm{Ab}$ & $2,4 \mathrm{~B} \mathrm{~cd}$ & $50,4 \mathrm{~A} a b$ & $0,0 \mathrm{~B} \mathrm{~b}$ & 4,0 A cde \\
\hline $30^{\circ} \mathrm{C}$ & $16,0 \mathrm{~B} \mathrm{a}$ & $48,0 \mathrm{~A} \mathrm{~b}$ & $2,6 \mathrm{~B}$ ab & $16,3 \mathrm{~A} \mathrm{~b}$ & $14,8 \mathrm{~A} \mathrm{a}$ & $9,1 \mathrm{~B} \mathrm{~b}$ & $10,4 \mathrm{~B}$ bc & $32,0 \mathrm{~A} \mathrm{bc}$ & $5,6 \mathrm{~B} \mathrm{a}$ & $16,0 \mathrm{~A} \mathrm{a}$ \\
\hline $35^{\circ} \mathrm{C}$ & $5,6 \mathrm{~A} \mathrm{~b}$ & $9,6 \mathrm{~A} \mathrm{~d}$ & $0,3 \mathrm{~B} \mathrm{~b}$ & $3,6 \mathrm{~A} \mathrm{c}$ & $15,3 \mathrm{~A} \mathrm{a}$ & $10,7 \mathrm{~A} \mathrm{~b}$ & $0,8 \mathrm{Ad}$ & $2,4 \mathrm{Ad}$ & $4,8 \mathrm{~A} \mathrm{a}$ & $7,2 \mathrm{~A} a b c$ \\
\hline $40^{\circ} \mathrm{C}$ & $0,0 \mathrm{~A} \mathrm{c}$ & $0,0 \mathrm{~A} \mathrm{e}$ & $0,0 \mathrm{~A} \mathrm{~b}$ & $0,0 \mathrm{~A} \mathrm{~d}$ & $0,0 \mathrm{~A} \mathrm{~b}$ & $0,0 \mathrm{~A} \mathrm{c}$ & $0,0 \mathrm{~A} \mathrm{~d}$ & $0,0 \mathrm{~A} \mathrm{~d}$ & $0,0 \mathrm{~A} \mathrm{~b}$ & $0,0 \mathrm{~A} \mathrm{e}$ \\
\hline $20-30^{\circ} \mathrm{C}$ & $23,0 \mathrm{~B} \mathrm{a}$ & $66,4 \mathrm{~A}$ a & $3,9 \mathrm{~B} \mathrm{a}$ & $27,6 \mathrm{~A} \mathrm{a}$ & $15,6 \mathrm{~A} \mathrm{a}$ & $10,4 \mathrm{~B} \mathrm{~b}$ & $20,8 \mathrm{~B} \mathrm{a}$ & $60,8 \mathrm{~A} \mathrm{a}$ & $2,4 \mathrm{~B}$ ab & $5,6 \mathrm{~A}$ bcd \\
\hline $30-20^{\circ} \mathrm{C}$ & $16,0 \mathrm{~B} \mathrm{a}$ & 49,6 A ab & $2,7 \mathrm{~B}$ ab & $17,4 \mathrm{~A} \mathrm{~b}$ & $14,5 \mathrm{~A} \mathrm{a}$ & $9,3 \mathrm{~B} \mathrm{~b}$ & $12,8 \mathrm{~B} \mathrm{ab}$ & $36,0 \mathrm{~A} \mathrm{bc}$ & $3,2 \mathrm{~B}$ ab & $13,6 \mathrm{~A} \mathrm{ab}$ \\
\hline \multicolumn{11}{|l|}{$\mathrm{F}$} \\
\hline Concentração & \multicolumn{2}{|c|}{$453 * *$} & \multicolumn{2}{|c|}{$333,3 * *$} & \multicolumn{2}{|c|}{$13,7 * *$} & \multicolumn{2}{|c|}{$279 * *$} & \multicolumn{2}{|c|}{$39,47 * *$} \\
\hline Temperatura & \multicolumn{2}{|c|}{$101 * *$} & \multicolumn{2}{|c|}{$38,5^{* *}$} & \multicolumn{2}{|c|}{$28,8^{* *}$} & \multicolumn{2}{|c|}{$65,5^{* *}$} & \multicolumn{2}{|c|}{$19,17 * *$} \\
\hline Conc.xTemp. & \multicolumn{2}{|c|}{$30,2 * *$} & \multicolumn{2}{|c|}{$19,11 * *$} & \multicolumn{2}{|c|}{$16,4^{* *}$} & \multicolumn{2}{|c|}{$20,29 * *$} & \multicolumn{2}{|c|}{$2,34^{*}$} \\
\hline C.V. (\%) & \multicolumn{2}{|c|}{21,6} & \multicolumn{2}{|c|}{25,1} & \multicolumn{2}{|c|}{25,8} & \multicolumn{2}{|c|}{30,3} & \multicolumn{2}{|c|}{66,39} \\
\hline
\end{tabular}

Médias seguidas de mesma letra, minúsculas na coluna e maiúsculas na linha, não diferem entre si pelo teste Tukey a 5\% de probabilidade.

* significativo a $1 \%$ de probabilidade.

** significativo a $1 \%$ de probabilidade.

Ns: não significativo a $5 \%$ de probabilidade.

Para o índice de velocidade de germinação (IVG), o maior valor $(27,6)$ foi obtido nas sementes submetidas à temperatura alternada $20-30^{\circ} \mathrm{C}$, diferindo significativamente das temperaturas constantes $20^{\circ} \mathrm{C}$ e $25^{\circ} \mathrm{C}(20,3$ e 21,7 ; respectivamente).

A eficiência dos reguladores vegetais no aumento da velocidade de germinação foi verificada também na variável tempo médio de germinação. Embora a porcentagem de germinação nas temperaturas de $15^{\circ} \mathrm{C}, 20^{\circ} \mathrm{C}$ e $25^{\circ} \mathrm{C}$ tenha aumentado após uso de $400 \mathrm{mg}$. $\mathrm{L}^{-1} \mathrm{de} \mathrm{GA}_{4+7}+\mathrm{N}$-(fenilmetil)aminopurina, o tempo médio de germinação não foi reduzido na mesma proporção. Porém, nas temperaturas de $30^{\circ} \mathrm{C}, 20-30^{\circ} \mathrm{C}$ e $30-20^{\circ} \mathrm{C}$, observou-se redução de 14,8 dias; 15,6 dias e 14,5 dias para 9,1 dias; 10,4 dias e 9,3 dias, respectivamente.
Para a porcentagem de plântulas normais, os resultados foram semelhantes à variável porcentagem de germinação, sendo as maiores médias obtidas em sementes tratadas com 400mg.L $\mathrm{L}^{-1}$ de $\mathrm{GA}_{4+7}+\mathrm{N}$-(fenilmetil)aminopurina e submetidas à temperatura de $20-30^{\circ} \mathrm{C}, 25^{\circ} \mathrm{C}$ e $20^{\circ} \mathrm{C}(61 \%, 50 \%$ e $42 \%$, respectivamente $)$.

$\mathrm{O}$ uso de 400mg.L $\mathrm{L}^{-1}$ de $\mathrm{GA}_{4+7}+\mathrm{N}$-(fenilmetil)aminopurina, combinado à temperatura de $30^{\circ} \mathrm{C}$ proporcionou as maiores médias de plântulas anormais (16\%), não diferindo dos tratamentos $400 \mathrm{mg} . \mathrm{L}^{-1}$ de $\mathrm{GA}_{4+7}$ $+\mathrm{N}$-(fenilmetil)-aminopurina, combinado às temperaturas de $30-20^{\circ} \mathrm{C}$ e $35^{\circ} \mathrm{C}(13,6 \%$ e $7,2 \%)$. Esse fato pode estar relacionado à disponibilidade de oxigênio, pois os autores Come e Tissaoui (1973 apud Cunha e Casali, 1989), ao estudarem a germinação de sementes de maçã, 
demonstraram que, em temperaturas acima de $25^{\circ} \mathrm{C}$, o oxigênio torna-se progressivamente menos solúvel em água e atinge o embrião em menores quantidades. Dessa forma, a menor ocorrência de plântulas anormais na temperatura 20$30^{\circ} \mathrm{C}$ pode estar relacionada à curta duração do período de temperatura alta, apenas 8 horas diárias a $30^{\circ} \mathrm{C}$, enquanto a alternância de $30-20^{\circ} \mathrm{C}$ permaneceu 16 horas diárias na maior temperatura.

A disponibilidade de oxigênio também pode estar relacionada às maiores porcentagens de sementes mortas observadas em temperaturas mais elevadas (Tabela 4), obtidas a partir de sementes tratadas com reguladores vegetais $\left(85,8 \%\right.$ e $92,8 \%$, nas temperaturas $35^{\circ} \mathrm{C}$ e $40^{\circ} \mathrm{C}$, respectivamente). Possivelmente, os reguladores vegetais foram suficientes para a superação do processo de dormência, no entanto o uso de temperaturas mais elevadas, associada à baixa disponibilidade de oxigênio, causou a morte das sementes.

TABELA 4. Sementes mortas e sementes dormentes de Passiflora cincinnata Mast. em função da temperatura e do pré-tratamento com $\mathrm{GA}_{4+7}+\mathrm{N}$-(fenilmetil)-aminopurina.

\begin{tabular}{|c|c|c|c|c|}
\hline & \multicolumn{2}{|c|}{ Sementes Mortas (\%) } & \multicolumn{2}{|c|}{ Sementes Dormentes $(\%)$} \\
\hline Temperatura & Água & 400mg. $\mathrm{L}^{-1}$ & Água & 400mg. $\mathrm{L}^{-1}$ \\
\hline $10^{\circ} \mathrm{C}$ & $8,8 \mathrm{~A} \mathrm{~b}$ & $12,8 \mathrm{~A} \mathrm{bc}$ & $91,2 \mathrm{~A} \mathrm{a}$ & $87,2 \mathrm{~A}$ a \\
\hline $15^{\circ} \mathrm{C}$ & $12,8 \mathrm{~A} \mathrm{~b}$ & $4,8 \mathrm{~A} \mathrm{c}$ & $87,2 \mathrm{~A} a b$ & $65,6 \mathrm{~B} \mathrm{~b}$ \\
\hline $20^{\circ} \mathrm{C}$ & $17,6 \mathrm{~A} \mathrm{ab}$ & $8,0 \mathrm{~B} \mathrm{c}$ & $80,8 \mathrm{~A}$ abc & $47,2 \mathrm{~B} \mathrm{bc}$ \\
\hline $25^{\circ} \mathrm{C}$ & $8,8 \mathrm{~A} \mathrm{~b}$ & $8,8 \mathrm{~A} \mathrm{c}$ & $88,8 \mathrm{~A}$ ab & $36,8 \mathrm{~B} \mathrm{~cd}$ \\
\hline $30^{\circ} \mathrm{C}$ & $17,6 \mathrm{~B}$ ab & $30,4 \mathrm{~A} \mathrm{~b}$ & $66,4 \mathrm{Ac}$ & $21,6 \mathrm{~B} \mathrm{~d}$ \\
\hline $35^{\circ} \mathrm{C}$ & $17,6 \mathrm{~B}$ ab & $85,80 \mathrm{~A} \mathrm{a}$ & $76,8 \mathrm{~A} \mathrm{bc}$ & $4,8 \mathrm{~B} \mathrm{f}$ \\
\hline $40^{\circ} \mathrm{C}$ & $34,4 \mathrm{~B}$ a & $92,8 \mathrm{~A} \mathrm{a}$ & $65,6 \mathrm{Ac}$ & 7,2 B ef \\
\hline $20-30^{\circ} \mathrm{C}$ & $14,4 \mathrm{~A} \mathrm{ab}$ & $14,4 \mathrm{~A} \mathrm{bc}$ & $62,4 \mathrm{~A} \mathrm{c}$ & $19,2 \mathrm{~B}$ de \\
\hline $30-20^{\circ} \mathrm{C}$ & $7,2 \mathrm{~B} \mathrm{~b}$ & $17,6 \mathrm{~A} \mathrm{bc}$ & $76,8 \mathrm{~A} \mathrm{bc}$ & $32,8 \mathrm{~B} \mathrm{~cd}$ \\
\hline \multicolumn{5}{|l|}{$\mathrm{F}$} \\
\hline Concentração & \multicolumn{2}{|c|}{$37,48 * *$} & \multicolumn{2}{|c|}{$388,4 * *$} \\
\hline Temperatura & \multicolumn{2}{|c|}{$35,97 * *$} & \multicolumn{2}{|c|}{$36,8 * *$} \\
\hline Conc.xTemp. & \multicolumn{2}{|c|}{$15,77 * *$} & \multicolumn{2}{|c|}{$9,85^{* *}$} \\
\hline C.V. (\%) & \multicolumn{2}{|c|}{29,36} & \multicolumn{2}{|c|}{13,58} \\
\hline
\end{tabular}

Médias seguidas de mesma letra, minúsculas na coluna e maiúsculas na linha, não diferem entre si pelo Teste Tukey a 5\% de probabilidade.

* significativo a $5 \%$ de probabilidade.

** significativo a $1 \%$ de probabilidade.

Os resultados obtidos para porcentagem de sementes dormentes corroboram os dados obtidos na porcentagem de germinação, demonstrando a eficiência dos reguladores vegetais $\mathrm{GA}_{4+7}+\mathrm{N}$-(fenilmetil)-aminopurina na superação da dormência das sementes. Observou-se que a aplicação de 400mg. $\mathrm{L}^{-1}$ de $\mathrm{GA}_{4+7}+\mathrm{N}$-(fenilmetil)-aminopurina, diminuiu acentuadamente a porcentagem de sementes dormentes à medida que aumentou a temperatura. Em sementes não tratadas (testemunha), essa diminuição não foi observada.

\section{CONCLUSÕES}

- A luz exerce efeito inibitório sobre a germinação de sementes de maracujá do mato.

- Os reguladores vegetais $\mathrm{GA}_{4+7}+\mathrm{N}$-(fenilmetil)aminopurina, nas concentrações de 300, 400 e $500 \mathrm{mg}$. $\mathrm{L}^{-1}$ são eficientes na superação da dormência das sementes.

- $\mathrm{O}$ uso de $\mathrm{GA}_{4+7}+\mathrm{N}$-(fenilmetil)-aminopurina na concentração de $400 \mathrm{mg}^{-1} \mathrm{~L}^{-1}$ amplia os limites de 
temperatura de germinação.

- A temperatura alternada de $20-30^{\circ} \mathrm{C}$ é a mais adequada para a germinação de sementes de Passiflora cincinnata Mast.

\section{REFERÊNCIAS}

ARAÚJO, F.P.; SANTOS, C.A.F.; LELO, F.M. Propagação vegetativa do maracujá do mato: espécie resistente à seca, de potencial econômico para agricultura de sequeiro. Petrolina, PE: Embrapa Semi-Árido, 2004. (Embrapa SemiÁrido. Instruções Técnicas, 61).

APONTE, Y.; JÁUREGUI, D. Algunos aspectos de la biología floral de Passiflora cincinnata Mast. Revista de la Facultad de Agronomia, v.21, n.3, p.211- 219, 2004.

BEWLEY, J.D.; BLACK. M. Seeds: physiology of development and germination. New York: Plenum Press, 1994. 445p.

BORGES, E.E.L.; RENA, A.B. Germinação de sementes. In: AGUIAR, I.B.; PINÃ-RODRIGUES, F.C.M.; FIGLIOGLIA, M.B. Sementes florestais tropicais. Brasília, DF: ABRATES, 1993. p.83-135.

BOTELHO, B.A.; PEREZ, S.C.J.G.A. Estresse hídrico e reguladores de crescimento na germinação de sementes de canafístula. Scientia Agrícola, v.58, n.1, p.43-49, 2001.

BRASIL. Ministério da Agricultura e Reforma Agrária. Secretaria Nacional de Defesa Agropecuária. Departamento Nacional de Produção Vegetal. Coordenação de Laboratório Vegetal. Regras para Análise de Sementes. Brasília, DF, $1992.365 \mathrm{p}$.

CUNHA, R.; CASALI, W.D. Efeito de substâncias reguladoras de crescimento sobre a germinação de sementes de alface (Lactuca sativa L.). Revista Brasileira de Fisiologia Vegetal, v.1, n.2, p.121-132, 1989.

DUARTE FILHO, J.; VASCONCELLOS, M.S.; CARVALHO, C.M.; LEONEL, S. Germinação de sementes de Pssiflora gibert N. E. Brown sob temperatura controlada. Revista Brasileira de Fruticultura, v.22, n.3, p.468-470, 2000 .

FERRARI, T. B. Germinação de sementes e análise de crescimento no estádio inicial do desenvolvimento de Passiflora alata Curtis com o uso de biorreguladores. 2005. 114 f. Dissertação (Mestrado - Botânica e Fisiologia Vegetal) - Instituto de Biociência, Universidade Estadual Paulista, Botucatu.

FERRAS GRANDE, F.G.A.; TAKAKI, M. Efeitos da luz, temperatura e estresse de água na Germinação de sementes de Caesalpinia peltophoroides Benth. (caesalpinoideae). Bragantia, v.65, n.1, p.37-42, 2006.

FERREIRA, F.R.; OLIVEIRA, J.C. Germoplasma de passiflora. In: SÃO JOSÉ, A.R. A cultura do maracujá no Brasil. Jaboticabal: FUNEP, 1991. p.187-200.

HADAS, A. Water uptake and germination of leguminous seeds under changing external water potential in osmotic solution. Journal of Experimental Botany, v.52, n.98, p. 480-489, 1976.

LABOURIAU, L.G. A germinação das sementes. Washington: OEA, 1983. 174p.

LOMBARDI, S.L. Estudos anatômicos e fisiológicos da organogênese in vitro em Passiflora cincinnata Mast. 2003. 60 f. Dissertação (Mestrado - Fisiologia e Bioquímica de Plantas), Escola Superior de Agricultura "Luiz de Queiroz", Universidade de São Paulo, Piracicaba.

MALAVASI, M.M.; FOGAÇA, C.A.; FOGAÇA, L.; FERREIRA, G. Preparo e coloração de sementes de maracujá doce (Passiflora alata Dryander) para avaliação da viabilidade através de teste do tetrazólio. Revista Brasileira de Fruticultura, v.23, n.1, p.126-129, 2001.

MELETTI, L.M.M.; FURLANI, P.R.; ALVAREZ, V.; SOARES-SCOTT, M.D.; BERNACCI, L.C.; AZEVEDOFILHO, J.A. Novas tecnologias melhoram a produção de mudas de maracujá. O Agronômico, v.54, n.1, p.30-33, 2002.

MORLEY BUNKER, M.J.S. Seed coat dormancy in Passiflora species. Annual Journal Royal New Zealand Institute of Horticulture, v.8, p.72-84, 1980.

OSIPI, E.A.F.; NAKAGAWA, J. Efeito da temperatura na avaliação da qualidade fisiológica de sementes do maracujádoce (Passiflora alata Dryander). Revista Brasileira de Fruticultura, v.27, n.1, p.179-181, 2005.

PIMENTEL GOMES, F. Curso de estatística experimental. Piracicaba: Nobel, 1990. 468p.

RUGGIERO, C. Enxertia do maracujazeiro. In: SÃO JOSÉ, A.R. A cultura do maracujá no Brasil. Jaboticabal: FUNEP, FCAVJ, Unesp, 1991. p. 43-59.

SANTOS, M.C.; SOUSA, G.R.L.; SILVA, J.R.; SANTOS, V.L.M. Efeito da temperatura e do substrato na germinação de sementes de maracujá (Passiflora edulis Sims. var. flavicarpa Deg.). Revista Brasileira de Sementes, v.21, n.1, p.1-6, 1999.

SÃO JOSÉ, A.R. Maracujá: produção e mercado. Vitória da Conquista: UESB, 1994. 255p. 
SILVA, J.B.C.; NAKAGAWA, J. Estudo de fórmulas para cálculos da velocidade de germinação. Informativo ABRATES, v.5, n.1, p.62-73, 1995.

ZUCARELI, C.; CASTRO, M.M.; OLIVEIRA, H.R.;
BRANCALIÃO, S.R.; RODRIGUES, J.D.; ONO, E.O.; BOARO, C.S.F. Fitoreguladores e germinação de sementes de maracujá doce em condições de laboratório. Scientia Agrária, v.4, n.1/2, p.9-14, 2003. 